قراءة في كتاب

\title{
المرأة والعمل السياسي: رؤية إسلامية
}

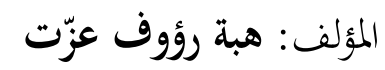

الناشر: المعهد العلمي للفكر الإسلامي، سلسلة الرسائل الجامعية، رقم18، الطبعة الأولى 1995م، (311)

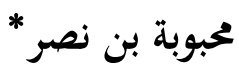

الكتاب هو دراسة قدمتها الباحثة المصرية الأستاذة هبة رؤوف عزت للحصول على درجة الماجستير

في العلوم السياسية من كلية الاقتصاد والعلوم السياسية بجامعة القاهرة سنة 1413هـ/1992م، وهي تتناول فيها مسألة مهمة جداً من المسائل المطروحة اليوم على الساحة الفكرية الإسلامية، ألا وهي قضية المرأة والعمل السياسي في الفكر الإسلامي.

يتكون الكتاب من 311 صفحة من الحجم المتوسط. وقد بنت الباحثة دراستها على مقدمة وثلاثة فصول وخاتمة، وقد احتوى كل فصل على عدد من المباحث، وتفرع كل مبحث إلى عدد من المطالب. وتضمن الكتاب كذلك ثبتاً بقائمة مهمة للمراجع العربية والإنكليزية احتلت تسعاً وثلاثين صفحة، فضلاً عن فهرس للأعلام وكشاف للموضوعات الواردة في الكتاب... وقد صدَّر للكتاب الدكتور طه جابر العلواني وقدم له المستشار طارق البشري.

دكتوراه في الفلسفة من جامعة باريس 10، 1414هـ/1993م، (وكان موضوع رسالتها: المرأة بين نسيان الفلاسفة وقميش 
إن أهمية الكتاب تكمن أولاً في موضوعه، إذ أنه موضوع اشتد حوله الجدل ودارت حوله نقاشات طويلة بين الإسلاميين والعلمانيين، وبين الإسلاميين أنفسهم، لا في أوساط الباحثين المختصين والعلماء والمثقفين فحسب، بل في كل الأوساط الاجتماعية، فهو موضوع ترتبط به وتتفرع عنه العديد من الإشكاليات الفكرية الأساسية في حياتنا الاجتماعية والسياسية.

وعلى الرغم من الجدل القائم حول هذا الموضوع منذ هاية القرن التاسع عشر مع مفكري عصر النهضة، فإن المرأة -صاحبة الشأن- ظلت غائبة غياباً فعلياً عنه.

وتتمثل الخاصية الثانية للكتاب في كونه من إنتاج قلم امرأة. أما الخاصية الثالثة فتتمثل في تجاوز المسائل التقليدية مثل التعليم والاختلاط... إلى طرح مسألة حيوية، هي الدور السياسي للمرأة في الرؤية الإسلامية، وهو من القضايا الشائكة داخل الفكر الإسلامي القديم والحديث، وتعد الدراسات التي خصصت لهذا الموضوع قليلة جداً. ونذكر على سبيل المثال كتاب سعيد الأفغاني "عائشة والسياسة"، الذي يخلص فيه الكتاب إلى تحريم المشاركة السياسية للمرأة، وكتاب فاطمة المرنيسي الذي تدرس فيه دور المرأة السياسي في مجتمع المدينة وهو بعنوان الحريم السياسي.

والإشكالية التي طرحتها الكاتبة وسعت إلى معالجتها، هي مشروعية العمل السياسي للمرأة في الرؤية الإسلامية من خلال النظر في النصوص الشرعية التأسيسية (القرآن والسنة) ونقد الفكر الإسلامي، إذ أن التراث الفقهي -في رأي الباحثة- "ليس إلا إنجازاً إنسانياً له شروطه الاجتماعية والثقافية والتاريخية... في

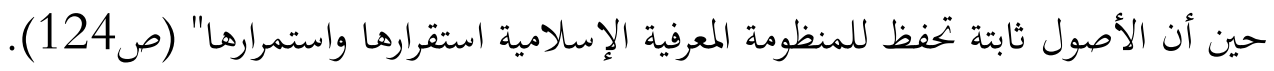
فإلى أي مدى استطاعت الدراسة تأصيل مشروعية العلم السياسي للمرأة، وصياغة منهجية معرفية إسلامية يمكن أنت تتنزل فعلاً في إطار مشروع "إسلامية المعرفة"؟.

في المقدمة تلخص الكاتبة مشروع البحث الذي طرحته على نفسها، وتحدد الإطار المعري والمبادئ التي تحكم هذا التصور وتمّيزهُ، فتتناول بالتحليل الرؤية الإسلامية لعلم المرأة السياسي في دائرتين: دائرة الأمة 
التي تشارك المرأة في فعالياتها السياسية بوصفها فرداً من أفرادها، ودائرة الأسرة التي تقوم في الرؤية الإسلامية بوظائف سياسية وتتحمل المرأة في إطارها مسؤوليات عديدة.

وتمدف هذه الدراسة - كما تؤكد الكاتبة- إلى بناء تصور كلي ونموذج معرفي للعمل السياسي للمرأة من منظور إسلامي يحدد الأبعاد المختلفة للموضوع وعناصره والعلاقات بينها، وفق مقاربتين متكاملتين على النحو الآتي:

1- أنه يربط بين ثلاثة بجالات، هي علم السياسة ودراسات المرأة والدراسات الإسلامية، فهو يهدف إلى صياغة خطاب إسلامي يكتوي هذه البمالات بشكل نقدي، ومن تم فهو نموذج تركبي. 2- أنه وإن كان يقوم بتحليل الدراسات المختلفة في هذه المجالات الثلاثة ونقدها ثم استنباط الرؤية الإسلامية، إلا أنه ليس مجرد بتريد لهذه العناصر، بل يضيف عناصر وأبعاداً جديدة تغيب عن التحليل السائد، ومن ثم فهو نموذج بتحيدي.

\section{أهمية الدراسة}

$$
\text { تتضح أهمية دراسة هبة رؤوف عزت على مستويين: }
$$

1- المستوى العلمي: إذ أها تتميز بطبيعتها المعرفية النظرية والإسلامية، وهو "ما يجعلها يختلفة عن الدراسات السابقة التي تناولت قضية العلم السياسي للمرأة، والتي لم تكن إشكاليتها قلة المادة المتاحة أو ضعف التحليل، بل غياب الرؤية المعرفية العلمية التي يتم في ضوئها دراسة المادة العلمية وتفسيرها. وهي إشكالية تنطبق على الدراسات الوضعية كما تنطبق على الدراسات الإسلامية (ص33).

فالكاتبة ترى أن الدراسات الإسلامية كانت تاريخية لا تتجاوز السرد إلى التحليل ثم التجريد، أو شرعية فقهية تدرس القضية من خلال آراء علماء السلف والترجيح بينها، ولا تتجاوز ذلك إلى محاولة التحديد والاجتهاد، أو قانونية تغفل الأبعاد الاجتماعية والسياسية. أما الدراسات العلمانية فقد تبنت 
المفاهيم الغربية وأسقطتها على المجتمعات الإسلامية دون أدنى مراجعة أو تقويه، وتناست الإطار المرجعي الذي يجب أن تحتكم إليه، في حين أن الدراسة التي بين أيدينا تمدف إلى بتحاوز هذه العقبات، فهي "تستند إلى الأصول الشرعية وتوظف المادة التاريخية وتستعين بالدراسات القانونية ولا تغفل الواقع، كما تحلل أبرز أطروحات دراسات المرأة وتقوّمها. هذه المنهجية في البحث، حسب تقدير الكاتبة، "مكنتها من تقديم خطاب جديد في مجالات علم السياسة ودراسات المرأة والدراسات الإسلامية" (ص32).

أما الأهمية العملية -كما تسميها الكاتبة- فتمثل في الموضوع نفسه بالنظر إلى أن "قضية المرأة قضية حيوية في الواقع الاجتماعي والسياسي للدول الإسلامية، وليست مجرد قضية بحث نظري وفكري. فقد أصبح كل ما يرتبط بالمرأة من قيم وتقاليد وأعراف وتشريعات ومؤسسات مجالاً للصراع الحضاري ومحكاً لاختبار عالمية المشروع العلماني في مقابل صلاحية المشروع الإسلامي وعالميته" (ص35). أما منهجية الدراسة فتؤكد الباحثة أها تتأسس على "أصولية المنهج"، أي ربط العقل بالوحي قرآنا وسنة دون التفريق بين العلوم كما فعل الفقهاء عندما صنفوا العلوم إلى "علوم شرعية" و "علوم نظرية فلسفية"، وعدوا "السياسة" من العلوم الفلسفية لا من العلوم الشرعية مدرجين مسائل كالإمامة والجهاد في أبواب الفقه. ولذا فإن "صياغة فقه سياسي ينبي على فهم للواقع وإدراكٍٍ لمقاصد الشرع، معتمداً في ذلك على قواعد علم أصول الفقه"، أمر كفيل بربط "السياسة كعلم اجتماعي إسلامي بالعلوم الشرعية بما يضمن تكامل العقل والوحي ويحقق لها الأصولية والتكامل مع العلوم الإسلامية الأخرى" (ص36).

إلا أن هذه العلاقة بالواقع لا تقف عند حدود الاستجابة لتطوره، بل تتجاوز ذلك إلى التأثير فيه وتقويمه انطلاقاً من مفهومي "الاستخلاف والأمانة"، وهذا ما تسميه الكاتبة "الاستقامة"، أي الارتباط بالقيم عند تحليل الظواهر الاجتماعية، والأخذ بالحسبان لمفاهيم العد والإيمان والشهادة، وبهذا تختلف عن الموضوعية التي تدعي استقلال العلم عن الأخلاق والقيم في العلوم الوضعية الغربية.

وبهذا المعنى فإن مفهوم الاستقامة يشير على أخلاقية العلم وضرورة أن تحكم عملية البحث مجموعة من القيم الأساسية كما أن الاستقامة تعني تقويم الواقع وفق الرؤية والمثالية الإسلامية، إذ أن "العلم ليس بجرد 
ممارسة عقلية نظرية بل وظيفة اجتماعية هدفها الإصلاح. وبهذا يختلف عن المنهج المعريز الغربي الذي يكتفي برصد الواقع ولا يدخل القيم في التحليل" (ص38).

حاولت الكتابة في الفصل الأول تحديد الإطار المعريف للرؤية الإسلامية للمرأة والعمل السياسي عبر

بيان المفاهيم الكلية التي تحكم التصور الإسلامي مثل مفاهيم التوحيد والاستخلاف، موضحة تميزها عن المفاهيم المقابلة لما في المنظومة المعرفية الغربية، كما تعرضت بالنقد للمفاهيم الأساسية التي تستخدم في دراسة العمل السياسي للمرأة مثل مفهوم "الدور" ومفهوم "حقوق المرأة" و "حقوق الإنسان"، و "المساواة"، مبينة المغالطة التي بُنيت عليها هذه التحليلات العلمانية في دراسة قضية المرأة، وداعية إلى الاستعاضة عن هذه المفاهيم بمفاهيم أخرى ترى أها أقدر على بناء منهج معرفي إسلامي، وإعطاء أبعاد عميقة لمكانة المرأة ووظيفتها في المجتمع. ومن هذه المفاهيم مفهوم العمل، والتوحيد والاستخلاف.

فالإنسان -انطلاقاً من مفهوم الاستخلاف - كائن مخلف تضبط حركته شريعة مصدرها الوحي ويرد إلى الله بعد الموت ليسأل عن أداء الأمانة والخلافة.

فمفهوم الخلافة أو الاستخلاف يعد مفهوماً أساسياً في إطار الرؤية الإسلامية لوظيفة المرأة حيث أن "استخلاف الإنسان" يشمل الرجال والنساء. وقد عبر القرآن عن هذا الشمول في آيات عديدة منها:

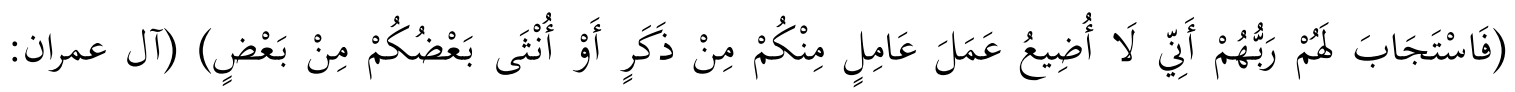

وتتمثل المساواة بين الرجال والنساء في المساواة في القيمة الإنساني والمساواة في الحقوق الاجتماعية وفي المسؤولية والجزاء. وهي المساواة التي تتأسس في جوانبها المختلفة على وحدة الأصل ووحدة المآل. وفي هذا السياق ترى الكاتبة أن الأحكام الخاصة بالنساء في القرآن -مثل إعفاء المرأة من الأعباء الاقتصادية للأسرة أو اختلاف نصيبها في الميراث- يُبقي استتناءات ترد على القاعدة التي هي المساواة" 
فالمساواة بين الرجل والمرأة في السياق القرآني هي -في رأي الكاتبة- "مساواة لها جوانبها المطلقة وجوانبها النسبية".

هذه هي النظرة الكلية انطلاقاً من المفاهيم الأساسية في الإسلام، وقد أدى غياها عند علماء السلف إلى فهم خاطئ لمكانة المرأة ووظيفتها. ففي علوم القرآن رأوا "أن النساء لا يدخلن في الخطاب القرآني إلا بقرينة بدلاً من أن يرون أهن يدخلن تغليباً في جمع المذكر السالم ولا يخرجن إلا بقرينة". كذلك في أصول الفقه فإن العلماء رأوا "أن النساء يدخلن في "الناس" و "القوم"، لكنهم عارضوا التغليب ما لم تدل عليه قرينة". كذلك فإن هذه الرؤية انعكست في كتب التفسير فوردت تفسيرات لبعض الآيات تضع المرأة في

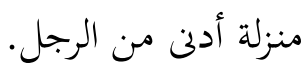
وترى الكاتبة أن هذه الرؤية الدونية للمرأة تأثرت "بتدهور وضع المرأة في بعض المجتمعات الإسلامية تبعاً لتدهور الاجتماعي والسياسي العام. إذ أن صريح النصوص القرآنية يخالفها بشكل قاطع" (ص60). وتنتقد الكاتبة هذه الرؤية التي تواصلت في الكتابات الإسلامية في العصر الحديث، تلك الكتابات التي اجتهدت في إثبات ضعف المرأة النفسي والعضوي من منطلق رد الفعل على المفهوم الغربي للمساواة الذي يلغي تماماً الفروق بين الرجل والمرأة.

وهنا يأتي واجب المفكر الإسلامي لتجاوز التطرف الحاصل من كلا الرأيين، باستخلاص مفهوم المساواة في الرؤية الإسلامية التي "تتميز بالوسطية" و المنضبطة "بمفاهيم التوحيد والاستخلاف" (ص26). وتنتقد الكاتبة الرؤية العلمانية لمفهوم المساواة بين الرجل والمرأة، التي ترتكز على المماثلة بين الله والإنسان والطبيعة، الأمر الذي أدى بالفكر الغربي إلى إعلان "موت الإله" مروراً باستنزاف الطبيعة وانتهاءاً بتدمير الإنسان، وأصبح الاحتكام إلى الواقع وتطور علاقات القوة بأشكالها المختلفة. وترى الباحثة أن الخلط والإخفاق في إدراك قيمة المساواة لم يكن على المستوى النظري والفلسفي فحسب، بل تعداه إلى الواقع حيث أن حركات "تحرير المرأة" تبنت هذه المفاهيم وانتقلت من "تحرير المرأة" إلى "النسوية" (Feminism) 
ممّا أدّى إلى تجاوز طلب المساواة إلى الصراع، ثم إلى تأكيد "الأفضلية النسوية"، ومن نقد "الأبوية" إلى طرح

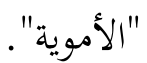

وتخلص الكاتبة بعد نقد العلمانية الغربية والعربية وبعض الكتابات الإسلامية إلى أن "أصولية المنهج الإسلامي والاحتكام للوحي هو الكفيل بتحقيق الاستمرارية والاستقامة والعالمية المتجاوزة للزمان والمكان. كما تؤكد أن السنن الكونية والفطرية للإنسان هي التي بتمع بين المجتمعات على اختلاف ثقافاتا، فترى أن الفكر الإسلامي ينقصه الوعي بهذه السنن و"خروج الأمة الإسلامية من عثرتا إنما عبر إدراك السنن التي تحكم أية قضية ومنها قضية المرأة" (صك) كوك).

هذه القضية يستلزم التعامل معها الجمع في الفقه والتطبيق بين السنن التشريعية الإلهية والسنن الاجتماعية والتاريخية. فلا بد من العودة إلى الأصول وفهم النصوص في ضوء مقاصد الشريعة التي بنيت على الفطرة، وهذا هو المنهج الذي يعصم من الفهم الجزئي للنصوص وتفسيرها تأثراً بواقع معين وتبني ممارسات خاطئة قد يغري نجاحها المؤقت بالاستمرار فيها. ومن هذا التكامل بين السنن التشريعية والسنن الاجتماعية، يكتسب الطرح الإسلامي صلاحه وصلاحيته.

هذه الفكرة التي حاولت الكاتبة تحليلها من أهم الأفكار الواردة في الكتاب إلا أن المشكل يبقى معلقاً. فكيف ننزل على مستوى الواقع هذه الفكرة النظرية التي طرحها في الحقيقة عدد من المفكرين المسلمين المعاصرين في إطار مشروع "بتديد أصول الفقه"، وهي إشكالية الربط بين النص والعقل والواقع لاستباط الأحكام. ولئن استطاع عدد من المفكرين صياغة الإشكالية إلا أن الأجوبة ظلت "عتشمة" تدور في إطار المنظومة الفقهية التقليدية، الأمر الذي جعل "الاجتهادات المعاصرة" لا تمارس التجديد إلا في بعض الجزئيات ولا تخرج في أحيان كثيرة من دائرة التوفيق والترجيح بين أقوال الفقهاء. تم تقدم الكاتبة تحليلاً لغوياً لبعض المفاهيم المستعملة في قضية المرأة مثل مفهوم "الدور" و "الحق" اللذين في إطارهما يحلل الفكر الغربي والعلماني بصفة عامة "الحقوق السياسية للمرأة" المتمثلة في حق الترشيح 
والانتخاب والوظائف العامة، فتلاحظ أن القانون العام في الفقه الإسلامي أقل تطوراً من القانون الخاص، وتعزو هبة عزت هذا القصور في القانون العام الإسلامي إلى الطبيعة الاستبدادية للحكومات المتعاقبة على الحكم في البلاد الإسلامية، وهي حكومات عمدت إلى إخماد حركة فقهية تقيم أصول الحكم على أسس من الحرية السياسية والحقوق العامة الديمقراطية. وقد نما القانون الخاص وتقدم تقدماً كبيراً لأن الحكومات المستبدة لم يكن يضيرها تقدمه.

وتذهب الكاتبة إلى أن تأصيل مفهوم "الحق" شرعياً في إطار القانون العام يواجه صعوبة شديدة، ولهذا يجب البحث عن مدخل أصولي آخر يمر عبر مفهوم "العمل" في الرؤية الإسلامية، ومفهوم "الواجب

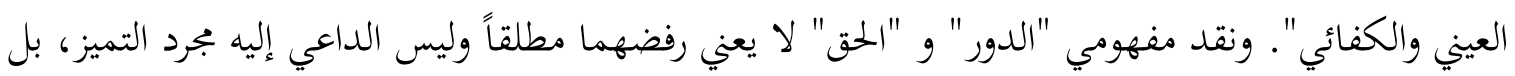
لأن هذين المفهومين أقل قدرة على التفسير والتحليل في إطار الفكر الإسلامي. فمفهوم "الدور" في الفكر الماركسي والنسائي أدى إلى إشكالية "صراع الأدوار"، والمرأة هي أشد الفئات تعرضاً لهذا الصراع، مما يثير نوعاً من الصراع الداخلي النفسي الحاد للتوفيق بين مختلف الأدوار. وبتنبّاً للسقوط في مأزق الصراع، ارتأت

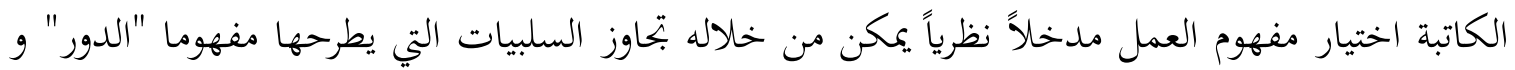
"الحق". وهي تعتقد أن مفهوم العمل أحد المفاهيم القرآنية الأساسية، وهو يرتبط بمفاهيم أساسية أخرى في المنظومة الإسلامية كالإممان والتقوى والاستخلاف... وقد جعله لله تعالى شرطاً من شروط الإسلام... وبنى التفضيل بين العباد والتفاوت في المنزلة لديه على العمل لا على الجنس أو النسب. وهنا تورد الكاتبة مختلف مراتب حكم العمل عند الأصوليين من حيث كونه واجباً أو مندوباً أو حراماً أو مباحاً أو مكروهاً. وأهم هذه المراتب في رأيها هو "الواجب" الذي هو إما واجب عيني أو واجب كفائي، وتدرج الكاتبة العمل السياسي في إطار الواجبات، وعلى هذا الأساس فالعمل السياسي "إما فرض عين كالبيعة العامة والشورى العامة، أو فرض كفاية كالجهاد والولايات العامة والأمر بالمعروف والنهي عن المنكر" (ص90).

وانطلاقاً من تعريف "السياسة" في أها "القيام على الشيء بما يصلحه... وإصلاح الخلق بإرشادهم إلى الطريق المنجي في الدنيا والآخرة، ولذا فهي تتسم بالعموم والشمول وتخاطب كل فرد:... فيرتبط مفهوم السياسة بالاستخلاف والتوحيد" (ص91)، فإن المرأة تدخل في هذا الخطاب، ويُصبح العمل السياسي 
مسؤولية فردية" وواجباً شرعياً لا ينفك عنه أحد من الناس إما على وجه العينية أو على وجه الكفاية، فهو

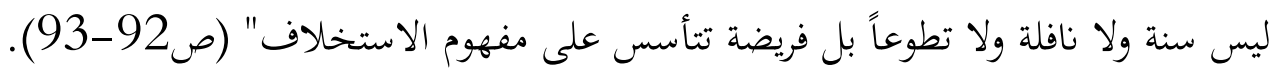

تم تبيّن الكاتبة الفرق بين مفهومي "الحق" و "الواجب"، والواجب لا يتوجه إلا إلى "القادر". وهنا

تكون التفرقة على أساس الأهلية والقدرة لا الجنس. ويصبح المقياس كسبياً إذ القدرة والأهلية يمكن امتلاكهما بالعمل، وبذلك يصبح التساؤل في بحث موضوع "المرأة والعمل السياسي" هو "أي المرأة؟" لا تعميم الأحكام بالحديث علن المرأة استبعاداً من الخطاب التكليفي أو إلزاماً به" (ص95).

أما الفصل الثاني فيسير على النسق نفسه تحليلاً لبعض المفاهيم وربطاً لها بمهمة الاستخلاف المنوطة بكل مسلم، و تأكيداً لمسألة الأهلية وأهمية الوعي وشروطه. وتتعرض الكاتبة بالنقد للموقف السائد بين الفقهاء نحو المرأة، وتناقش مفهوم "التقسيم الاجتماعي للعمل" الذي على أساسه يرى بعضطم أن المرأة مجالها البيت والأسرة، بينما يتحمل الرجل مسؤولية العلم الاقتصادي والسياسي. وعلى الرغم من إقرار كتابات هؤلاء بأن المساواة بين النساء والرجال في الحقوق والواجبات هي الأصل، إلا أن السائد هو "تفضيل عدم زج المرأة بنفسها في السياسة". بل ذهب بعضهم إلى حد القول "بأن السياسة على المرأة حرام صيانة للمجتمع من التخبط وسوء المنقلب". ولذا فإن طرح قضية العمل السياسي في ظل هذا الواقع الفكري للمجتمعات المسلمة يستلزم إعادة النظر في المسلمات التي بني عليها ذلك الواقع، ويصبح التركيز على مسألة الأهلية شرطاً ضرورياً لأي بحث في هذه القضية.

وحتى تكتسب المرأة الأهلية، تدعو الباحثة إلى أن تؤم النساء المؤسسات الاجتماعية التي تساعد

$$
\text { الفرد على الوعي بالظروف العامة للحياة ثقافياً وسياسياً واجتماعياً... إلخ. }
$$

وهنا تنبه الكاتبة إلى مواطن التناقض في آراء الفقهاء الذين يرون أن المرأة لها الأهلية الكاملة في الولاية الذاتية والمتعدية على الأموال والولاية المدنية (كالحضانة والوصاية)، في حين يتحفظون على أهليتها لممارسة العمل السياسي. 
وقد استند هذا الرأي على آيتئ "الشهادة" و "القوامة" وبعض الأحاديث النبوية. فتحدث بعضطم عما طبع عليه النساء من نقص واعوجاج في أخلاقهن وميلهن إلى إتباع الهوى في مقابل التفوق الطبيعي في استعداد الرجال" (ص101) (101).

تم تحاول الكاتبة بيان مستويات الشهادة وكيف أن القرن لم يخصص إلا في آية "الدين"، إلا أن هذا لم يمنع الفقه التقليدي من الخلط في هذه المسألة. وبحدر الإشارة هنا إلى أن الكاتبة على الرغم من محاولتها الجمع بين كل الآيات في مسألة الشهادة، إلا أها بقيت على مستوى الترجيح والتوفيق بين آراء الفقهاء قديمهم وحديثهم، متناسية المطلب الأساسي الذي انطلقت منه وهو النظرة الشمولية في القراءة المعرفية والبحث عن "العلة" في الحكم. كما أنها لم تتعرض إلى محاولة الشيخ محمد عبده والشيخ محمد شلتوت اللذين حاولا تطوير فهم متجلد في مسألة الشهادة انطلاقاً من علة الحكم في آية الدين واعتبار القاعدة الفقهية"الحكم يدور مع العلة عدماً ووجوداً، وإن اقتصر عملهما على هذه القضية ولم يتجاوزاها لتطوير منهج جديد في استباط الأحكام.

فالعمل السياسي، في رأي الكاتبة لا يرتبط بالقانون بل بالشريعة، ولا يلتزم بالمؤسسة بل بالمصالح الشرعية، وهذا ما يضفي على لمفهوم العلم السياسي أبعاداً تختلف عن "المشاركة السياسية". وأبرز مظهر من مظاهر العمل السياسي يتجلى في "البيعة"، إذ هي التي تضفي الشرعية على نظام الحكم، ولا تنتهي المسؤولية بعقد البيعة بل تستمر من خلال المشاركة في الشورى والرقابة على الحاكم ونصحه إذا حاد وعزله إذا لزم الأمر. وتتعرض الكاتبة عند بحث مفهوم البيعة إلى التقسيمات الفقهية، وتنقد المواقف التي ركزت على بُعد الطاعة من جانب الرعية دون التفصيل في التزام الحاكم.

وترى هبة عزت أن المرأة تستمد شرعية العمل السياسي انطلاقاً من واجب البيعة. فهناك بيعة عينية واجبة على كل مسلم ومسلمة، وهي البيعة على العقيدة والأخلاق، وقد سُميت اصطلاحاً "بيعة النساء". وهناك البيعة الكفائية التي هي واجبة على خاصة المسلمين دون عامتهم، وهي المرتبطة بالأمور الكفائية كالبيعة على الجهاد أو الولايات العامة. وقد أدى اصطلاح بيعة النساء إلى التفرقة بين بيعة النساء وبيعة 
الرجال، مما نتج عنه عزل المرأة عن الأمور العامة، وينطوي هذا التقسيم على الخلط بين البيعة العينية والبيعة الكفائية وافتراض نقص أهلية المرأة.

وتبيّن الباحثة اختلاف الآراء الفقهية في هذا المجال، وهو اختلاف نتج عن الاختلاف في فهم النصوص قرآناً وسنّة، مثل آية القوامة التي رأى بعضهم أها تتعدى المجال الأسري إلى المجال العام، والشهادة في الأموال التي سحب حكمها على بقية مجالات الشهادة. ومهما كان من أمر هذا الاختلاف، إلا أن هناك إجماعاً على عدم تولية المرأة الولاية الكبرى، وقد اعتمد هذا المنع على حديث "لم يفلح قوم ولّوا أمههم امرأة" وحديث "النساء ناقصات عقل ودين". تعتقد الكاتبة أن هذا الاختلاف في الآراء يرجع أساساً إلى عدم النظرة الشمولية للأصول الإسلامية.

تم تورد الكاتبة مفهوماً آخر بوصفه أحد مداخل قضية العمل السياسي، ولعله المفهوم الأكثر حيوية وشرعية، وهو مفهوم الجهاد. فالجهاد يصبح حاجة أكيدة في ظل الدولة القطرية العلمانية التي أحدثت انفصاماً بين العقيدة والدولة، وأرست مؤسسات حكومية علمانية تسيطر على كل قطاعات المجتمع. وهنا تأتي ضرورة "الجهاد" و "الخروج" لمواجهة هذه الأنظمة التي ابتعدت عن الشرع.

وبالنظر إلى الجهاد المسلح في هذه الحالة غير ممكن نظراً لطبيعة المؤسسات الحديثة، وبطش الدولة القطرية وقوتا، فإن هذا الواجب لا يسقط بل يأخذ أشكالاً يختلفة تتفق وظروف الواقع الراهن الذي تمر به الأمة. ونظراً للطبيعة الهيكلية للمجتمع، فإن العمل يجب أن يكون عبر تحرير الأبنية والمؤسسات. فالجهاد هذا الشكل ينبغي أن يكون تربوياً وإعلامياً واقتصادياً وثقافياً. وهذا يتطلب نفيراً عاماً لكل القدرات نساءً

أما "الخروج" فيمكن أن يأخذ عدة أشكال، أولها الامتناع عن المشاركة في المساندة أو التأييد للنظام القائم. وعلى الرغم من السياق الاجتماعي والعرف اللذين يقيدان حركة المرأة، إلا أن مدخل الجهاد والخروج يمكن أن يؤدي -في رأي الباحثة- إلى قبول التغيير دون تصادم مع "الرأي العام" المحافظ، إذ يصبح الواقع أكثر مرونة في ظل المناخ الجهادي، وهو ما يمكن أن يساعد على قبول المواقف الجديدة بشأن مكانة المرأة ووظيفتها في المجتمع ويكسبها الشرعية. 
وتختم الكاتبة بحثها بتحليل مطول لمقام الأسرة في التغيير الاجتماعي، وبالتالي وظيفة المرأة من خلال هذه الوحدة الأساسية للمجتمع، وهو م تناولته في الفصل الثالث من الكتاب.

ترى هبة عزت أن الأسرة يجب أن تكون وحدة التحليل في مختلف العلوم الإنسانية، إذ أن الرؤية الإسلامية لا تفرق بين العام والخاص، وذلك من خلال تحليل لفلسفة الأسرة في الإسلام -التي تختلف عنها في الفكر الغربي- ومسؤوليتها في التنشئة الاجتماعية والثقافية خاصة في ظل أزمة الدولة العطرية في العالم الإسلامي، التي تخلت عن دورها الحقيقي للتنشئة.

وهذا البحث في وظيفة الأسرة يجر الكاتبة إلى البحث في الأسرة الممتدة وما يمكن أن تضطلع به في إطار عملية التغيير. فهي ترى أفها وحدة ضرورية لمواجهة المؤسسات الاجتماعية النظامية، خاصة أن الأسرة هي الوحدة الاجتماعية الوحيدة التي لم تخترقها السيطرة المباشرة للأنظمة، وهذا ما "يرشحها لأن تكون أقوى جبهات التنشئة الحضارية ولمقاومة الدولة العلمانية" (ص233). فالأسرة الممتدة يمكن أن تكون إطاراً صالحاً من خلال مقاطعة البضائع الأجنبية والقيام بعمليات إنتاجية (زراعية وحرفية). ومن ثم فإنه بإمكاها أن

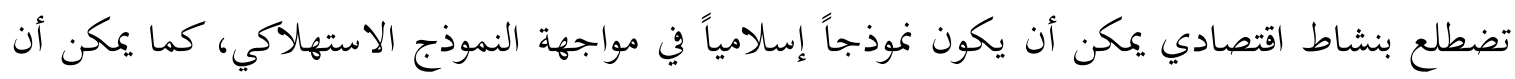
تكون إطاراً صالحاً للقيام بوظيفة قضائية بين أفرادها، فتوفر بذلك إطاراً مناسبا لتطبيق الشريعة في المعاملات الاجتماعية والاقتصادية وتحتل الشريعة مساحة العرف إلى أن تتمكن من العودة لدائرة القانون.

كذلك، في مجال التعليم، يمكن للأسرة من خلال مسؤوليتها في التنشئة مقاطعة المؤسسات التعليمية، وصياغة رؤية عن طريق الكتاتيب وحلقات التوعية والتثقيف. ولدعم هذه الرؤية للأسرة تورد الكاتبة مثال الأسرة الفلسطينية وإسهامها في مقاومة الاحتلال اليهودي، وكيف أن كافة أنشطة الانتفاضة لم تلجأ للمجابهة المباشرة مع العدو بل إلى استراتيجية يمكن تسميتها "التصعيد الأفقي". وبقدر ما أثرت الأسرة الممتدة في قيام الانتفاضة واستمرارها، فقد أثمرت تغييراً في طبيعة مسؤوليات المرأة داخل الأسرة وخارجها. - مان. 
وتخلص الكاتبة إلى أن هذه التجربة يمكن أن تتجاوز الخصوصية الفلسطينية لتصبح تخربة للمشروع

$$
\text { الحضاري الإسلامي. }
$$

وهذا التحليل لوظيفة الأسرة والرجوع إلى الأسرة الممتدة يبدو لنا مقترحاً غير واقعي، إذ تناست الكاتبة التعقيد الذي تتسم به المؤسسات في المجتمعات الحديثة والقوانين التي تربط الفرد بالدولة، ويكتسب من خلالها حقه في المواطنة من أبسط الإجراءات مثل تسجيل الولادات وعقود الزواج والوفيات إلى الدولة ومع المؤسسات الاقتصادية العملاقة. وبهذا فإن هذا الفصل وإن بعمق واقع الأسرة الفلسطينية، إلا أنه يتناقض مع ما أوردته الكاتبة في فصل آخر عندما تحدثت عن ضرورة أن تؤم المرأة (والرجل) المؤسسات الاجتماعية، والتعليمية والثقافية بوصفها الساحة التي يتفاعل فيها أفراد المجتمع، والعمل عل تغييرها من الداخل دون تصادم مع النظام القائم، وهذا يفترض وعياً عميقاً بالمناهج التربوية والثقافية للنظام السياسي والاجتماعي السائد حتى تتمكن قوى التغيير م استيعاجما ثم تقويضها وتطوير البديل.

ولعل هذا الرأي الذي ذهبت إليه الكاتبة في ذلك الفصل أقرب إلى الواقع. إذ الانطواء داخل الأسرة في وقتنا الراهن نوع من الهروب من مواجهة الواقع وعدم فهم للآليات التي تحكم المجتمعات وارتباطها ببقية الثقافات مع التقدم (التكنولوجي). إلا أن قصور هذا الفصل لا ينقص من أهمية الكتاب الذي وإن تعددت مقالاته وتنوعت أبوابه وفصوله، فإنه يربط بينها هدف واحد وهو إيجاد منهجية لفهم النص قرآناً وسنّة، ومراجعة الفقه الإسلامي من خلال موضوع "العمل السياسي للمرأة". ولئن انصب جهد الكاتبة على تحليل مفاهيم مثل الشورى والبيعة والجهاد، إلا أن دراستها في أبواب مختلفة شتت من وحدة هذه المفاهيم، التي كان يمكن أن تكوّن أساساً معرفياً لاستخلاص المنطق الداخلي للنص، تحدد من خلاله المنظومة القانونية التي تحكم حقوق المرأة وواجباها بعيداً عن مقاربة التوفيق والترجيج. وهنا نستعيد السؤال الذي طرحناه في بداية تقديم هذا الكتاب: "إلى أي مدى استطاعت تأصيل مشروعية العمل السياسي للمرأة وصياغة منهجية معرفية تتنزل في إطار مشروع "إسلامية المعرفة". ثم إلى أي مدى استطاعت الكاتبة التخلص من الفقه التقليدي والأساس النظري الذي انبنى عليه؟ (إذ لم تفتأ الباحثة في دراستها ترجع إلى التعريفات الأصولية التقليدية). فهل لا يمكن لفقيه اليوم أن يؤصل نظرية معرفية إلا 
مروراً بما حدده الأوئل في علوم القرآن وعلوم الحديث وأصول الفقه؟ أم أن بناء نظرية معرفية جديدة لا يستلزم مراجعة للآراء الفقهية الموروثة والترجيح بينها فحسب، وإنما بناءاً معرفياً يتناسق مع مشروع بتحيد

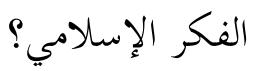

أما عن مشروعية العمل السياسي للمرأة، فيبدو لنا أن الباحثة بقيت فيها -إلى حد كبير - حبيسة الأطر والقوالب الفكرية التقليدية، ولم تستطع أن تعالج الموضوع في إطار منهجية معرفية كما وعدت في مقدمة الكتاب، وذلك بسبب اعتمادها على التفرقة بين الحق والواجب واعتبار الواجب المدخل الوحيد لفرض وظيفة جديدة للمرأة في المجتمع في الرؤية الإسلامية. فالفكرة التي تبقى في ذهن القارئ هي أن المرأة لا يمكن أن تساهم في مؤسسات المجتمع إلا من باب "فقه الواجب والضرورة". فما هي مشروعية مساهمة المرأة في حالة قيام الدولة الإسلامية؟

وهنا نرى ربط المفاهيم بعضها ببعض والنظر إلى تداخلها وتكاملها، حيث لا يتضارب مفهوم العدل مع المساواة، ولا الحق مع الواجب، فالعلم انطلاقاً من هذه الرؤية هو حق من حقوق المرأة حسب أهليتها وميولاتما ويمكن أن يصبح واجباً في ظروف طارئة أو في اختصاصات معينة.

وعل الرغم من بعض السلبيات التي أشرنا إليها، فأهمية الكتاب لا تكمن في ما دافعت عنه الكاتبة من مواقف، ولكن في محاولة تفكيك المفاهيم، والتفرقة بين الأصول والتراث الفكري. فقيمة الكتاب لا تكمن فيما قدمته من إجابات بقدر ما تكمن في طرح السؤال وفتح الفكر الإسلامي على مسألة شائكة منه تنبع وإليه تعود، والدعوة إلى تجاوز الانفصام الفكري الذي يعيشه العقل المسلم عبر طرح المسائل طرحاً صحيحاً وإن تأخرت الأجوبة. 